\title{
Change point detection in process control with robust individuals control chart
}

\author{
Kooi Huat $\mathrm{Ng}^{1 *}$, Kok Haur $\mathrm{Ng}^{2}$, and Jeng Young Liew ${ }^{3}$ \\ ${ }^{1}$ Department of Mathematical and Actuarial Sciences, Lee Kong Chian Faculty of Engineering and \\ Science, Universiti Tunku Abdul Rahman, Jalan Sungai Long, Bandar Sungai Long, Cheras, 43000 \\ Kajang, Selangor, Malaysia \\ ${ }^{2}$ Institute of Mathematical Sciences, Faculty of Science, University of Malaya, 50603 Lembah Pantai, \\ Kuala Lumpur, Malaysia \\ ${ }^{3}$ Department of Agro-Based Industry, Faculty of Agro-Based Industry, Universiti Malaysia Kelantan, \\ Jeli Campus, 17600 Jeli, Kelantan, Malaysia
}

\begin{abstract}
It is crucial to realize when a process has changed and to what extent it has changed, then it would certainly ease the task. On occasion that practitioners could determine the time point of the change, they would have a smaller search window to pursue for the special cause. As a result, the special cause can be discovered quicker and the necessary actions to improve quality can be triggered sooner. In this paper, we had demonstrated the use of so-called exploratory data analysis robust modified individuals control chart incorporating the M-scale estimator and had made some comparisons to the existing charts. The proposed modified robust individuals control chart which incorporates the M-scale estimator in order to compute the process standard deviation offers substantial improvements over the existing median absolute deviation framework. With respect to the application in real data set, the proposed approach appears to perform better than the typical robust control chart, and outperforms other conventional charts particularly in the presence of contamination. Thus, it is for these reasons that the proposed modified robust individuals control chart is preferred especially when there is a possible existence of outliers in data collection process.
\end{abstract}

\section{Introduction}

Control charts are useful devices employed to detect uncommon behavior in the manufacturing process. Apart from the use in the setting of statistical process control, they are also applied as an exploratory tool in data analysis. It is common to see that monitoring a process under contamination via conventional charting procedure such as Shewhart's $X$-bar or $\bar{X}$ chart and range, $R$ chart may cause undesirable number of false alarms [1]. Thus, robust control chart is an alternative tool for the conventional charts that maybe contaminated. This is due to the downweighting of the influences of the outlying data values [2]. In this study, we have presented a charting framework that satisfies the requirements of exploratory

*Corresponding author: khng@utar.edu.my 
analysis in the retrospective phase I, which has the intention to detect the assignable causes by unfolding the sign of variations.

In essence, the development of statistical process control charts has its purpose to aid practitioners to monitor if the change has developed [3]. In the event that a change does appear, the chart should react as soon as possible, and serves as a tool to detect for the process changes to tell apart between the special causes of variation and the common causes of variation. As long as the control chart statistics fall within its limits, the control chart suggests that only the common causes of variation exist. In contrast, when the chart statistics exceeds the limits, the control chart exhibits that there might be one or more special causes existed. One of the important criteria for assessing the performance of a control chart performs is how quickly it responds to the changes. The quicker a process change is discovered by the chart, the quicker process engineers can start their inspection for the special cause. The necessary action can then be taken to correct the process once it has been diagnosed.

Various groups of researchers have worked with some relevant robust control charts. For example, [4] had written widely about robust multivariate control chart in relation to goodness-of-fit test while [5] were investigating the concept of robust multivariate EWMA control chart concerning the sparse mean shifts. [6] have discussed a stepwise approach to construct a robust Shewhart location control chart and [7] has done extensive work with frameworks of automating phase I process that affect the phase II performance for individuals control chart.

\section{M-Scale estimate}

To fix the ideas of a robust M-scale estimate, [8] defined the scale M-estimates by letting $\rho(\cdot)$ to be a real function, satisfying the following assumptions.
(i) $\rho(0)=0$;
(ii) $\rho(-u)=\rho(u)$;
(iii) $0 \leq u \leq v$ implies $\rho(u)=\rho(v)$;

(iv) $\rho(\cdot)$ is continuous; (v) let $a=\sup \rho(u)$, then $0 \leq a \leq \infty$;

(vi) $\rho(u) \leq a$ and $0 \leq u<v$, then $\rho(u)<\rho(v)$.

More specifically, for a sample of size $n, u=\left(u_{1}, u_{2}, \ldots, u_{n}\right)$, the scale estimate $s(u)$ is evaluated through the value of $s$, which is the solution of the equation

$$
(1 / n) \sum_{i=1}^{n} \rho\left(u_{i} / s\right)=b
$$

where $b$ is defined to be $\mathrm{E}_{\varphi}(\rho(u))=b$, in which, $\varphi$ represents the standard normal distribution. Also, it is not difficult to verify that if

$$
c(u)=\left[\#\left\{i: 1 \leq i \leq n, u_{i}=0\right\} / n\right]<[1-(b / a)],
$$

then Equation (1) possesses a unique solution and the solution is different from 0. Suppose that $c(u) \geq 1-(b / a)$, we define $s(u)=0$.

In practice, the M-scale estimate is computed via the implementation of the following two stages:

Stage 1: Calculate the high breakdown-point location estimate $T_{0, n}$ of $\theta_{0}$, presumably 0.5 . Herein median is adopted as an initial estimate.

Stage 2: Compute the residuals

$$
r_{i}\left(T_{0, n}\right)=y_{i}-T_{0, n}, \quad \text { for } \quad 1 \leq i \leq n,
$$


and evaluate the M-scale $s_{n}=s\left(r\left(T_{0, n}\right)\right)$ given by Equation (1). For which the appropriate function of $\rho(\cdot)$ is chosen so as to satisfy the assumption (A). While the constants $a$ and $b$ are determined in such a way that

$$
b / a=0.5,
$$

where $a=\max \rho(u)$. As proved by [8], Equation (4) implies that the scale estimate has breakdown-point of 0.5 . The objective function $\rho(\cdot)$ is found by integrating Hampel's redescending $\psi_{H}$ function denoted by $\rho_{H}$ ([9]) with $(a, b, c)=(1.5,3.5,8)$ as indicated by Fig. 1. And that $\rho_{H}(u)$ can be obtained via $\rho_{H}(u / k)$ ([10]). Through numerical integration, by taking $k=0.212$, it appears to show that the scale estimate has a high breakdown of 0.5 . [11] defined the derivative of $\rho_{H}$ as

$$
\psi_{H}(t)= \begin{cases}t, & \text { if }|t|<a \\ a \operatorname{sgn}(t), & \text { if } a \leq|t| \leq b \\ \{(c-|t|) /(c-b)\} a \operatorname{sgn}(t), & \text { if } b \leq|t| \leq c \\ 0, & \text { otherwise, }\end{cases}
$$

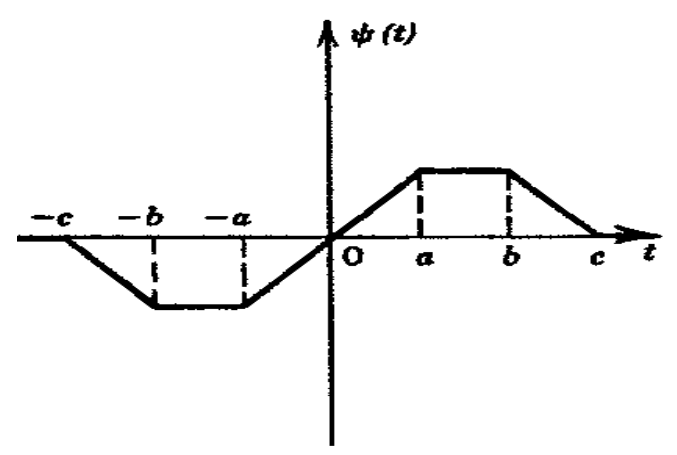

Fig. 1. Hampel-type $\psi$-function [12].

which is called a three-part redescending M-estimator to safeguard against extreme outlying observations.

\section{De Mast and Roes control-charting procedure}

[13] proposed the series of steps for the control-charting framework which is demonstrated as follows:

1. Initially, determine the locations of potential shifts and initiate the significance test using the test statistic $R T^{2} / n_{1}$. Then, the current data is partition into the corresponding intervals.

2. Once the completion of this step, compute the means between the intervals of the subsequent shifts as well as the variance of in-control measurements using robust estimators.

3. Employing the estimates, set up a pair of control limits for each interval. Observations are deemed to be outlying values if they lie outside the control limits. 
For illustrative purposes, presuming that the null model of the in-control process with the measurements $y_{i}, \quad i=1,2, . ., n$, possessing an identical normal distribution,

$$
y_{i}=\mu_{j}+\varepsilon_{i}, \quad \text { for } \quad i=1,2, \ldots, n ; j=1,2,
$$

with $\mu_{j}$ is population mean and $\varepsilon_{i}$ is independently and identically distributed $\mathrm{N}\left(0, \sigma^{2}\right)$. To obtain the corresponding estimations, let us begin looking at the way to estimate the shift position, $\tau$, assuming that a single shift does occur. Such scenario is demonstrated as below:

$$
\left\{\begin{array}{lll}
y_{i}=\mu_{1}+\varepsilon_{i} & \text { for } & i=1,2, \ldots, \tau, \\
y_{i}=\mu_{2}+\varepsilon_{i} & \text { for } & i=\tau+1, \tau+2, \ldots, n .
\end{array}\right.
$$

Here, $\mu_{1}, \mu_{2}, \sigma$ and $\tau$ are to be estimated. [14] proposed the maximum likelihood (ML) estimator for $\tau$. As to the ML estimators for $\mu_{1}, \mu_{2}$, and $\sigma$, together with $\tau$, they are described as

$$
\left(\tilde{\mu}_{1}, \tilde{\mu}_{2}, \tilde{\sigma}, \tilde{\tau}\right)=\arg \min _{\mu_{1}, \mu_{2}, \sigma, \tau} l\left(\mu_{1}, \mu_{2}, \sigma, \tau\right),
$$

with

$$
l\left(\mu_{1}, \mu_{2}, \sigma, \tau\right)=\frac{n}{2} \log \left(2 \pi \sigma^{2}\right)+\frac{1}{2 \sigma^{2}}\left(\sum_{i=1}^{\tau}\left(y_{i}-\mu_{1}\right)^{2}+\sum_{i=\tau+1}^{n}\left(y_{i}-\mu_{2}\right)^{2}\right) .
$$

The term arg min $l(x)$ represents the $x$ value where $l(x)$ is to be minimized. In order to determine $\tau$, perform the partial derivatives for $\mu_{1}, \mu_{2}$ and $\sigma$, and solving the respective equations by equating to zero. The resulting estimators for fixed $\tau$ are as below:

$$
\begin{gathered}
c \tilde{\mu}_{1}[\tau]=\frac{1}{\tau} \sum_{i=1}^{n} y_{i}, \\
\tilde{\mu}_{2}[\tau]=\frac{1}{n-\tau} \sum_{i=\tau+1}^{n} y_{i}, \\
\tilde{\sigma}^{2}[\tau]=\frac{1}{n}\left(\sum_{i=1}^{\tau}\left(y_{i}-\tilde{\mu}_{1}[\tau]\right)^{2}+\sum_{i=\tau+1}^{n}\left(y_{i}-\tilde{\mu}_{2}[\tau]\right)^{2}\right), \\
\tau=2,3, \ldots, n-2 .
\end{gathered}
$$

Then,

$$
\tilde{\tau}=\arg \min _{2 \leq \tau \leq n-2}\left(\tilde{\mu}_{1}[\tau], \tilde{\mu}_{2}[\tau], \tilde{\sigma}[\tau], \tau\right) .
$$

Consequently,

$$
\tilde{\mu}_{1}=\tilde{\mu}_{1}[\tilde{\tau}], \quad \tilde{\mu}_{2}=\tilde{\mu}_{2}[\tilde{\tau}], \quad \text { and } \quad \tilde{\sigma}=\tilde{\sigma}[\tilde{\tau}]
$$

In addition to this, it is possible that not all observations are stemmed from the in-control Equation (6) without fail. Suppose that there are some observations (outliers) might be coming from a non-normal or a heavy-tailed distribution. To ensure the framework more robust in the presence of outlying values, it was adjusted accordingly, in which, with $\tau=2,3, \ldots, n-2$, let $\hat{\mu}_{1}[\tau]$ and $\hat{\mu}_{2}[\tau]$ satisfy the following equation

$$
\sum_{i=1}^{\tau} \psi\left(\frac{\left(y_{i}-\mu_{1}\right)}{c s_{0}[\tau]}\right)=0 \text { and } \sum_{i=\tau+1}^{n} \psi\left(\frac{\left(y_{i}-\mu_{2}\right)}{c s_{0}[\tau]}\right)=0,
$$

where

$$
s_{0}[\tau]=\operatorname{median}\left\{\left|y_{i}-m\right|\right\}_{i=1,2, \ldots, n},
$$


with $m=m_{1}[\tau]$ for $1 \leq i \leq \tau$, and $m=m_{2}[\tau]$ for $\tau+1 \leq i \leq n$, for which $m_{1}[\tau]$ and $m_{2}[\tau]$ are the associated medians values for $y_{1}, y_{2}, \ldots, y_{\tau}$ and $y_{\tau+1}, y_{\tau+2}, \ldots, y_{n} . \psi$ denotes an odd function with $c$ is a tuning constant.

Taking $s_{0}[\tau]$ as initial estimate for scale, $\hat{\mu}_{1}[\tau]$ and $\hat{\mu}_{2}[\tau]$ are M-estimators for the location. The final scale estimate can be described by

$$
\hat{\sigma}^{2}[\tau]=\frac{\sqrt{n} c s_{0}[\tau]\left(\sum_{i=1}^{\tau} \psi^{2}\left(\left(y_{i}-\hat{\mu}_{1}[\tau]\right) / c s_{0}[\tau]\right)+\sum_{i=\tau+1}^{n} \psi^{2}\left(\left(y_{i}-\hat{\mu}_{2}[\tau]\right) / c s_{0}[\tau]\right)\right)^{1 / 2}}{\left|\sum_{i=1}^{\tau} \psi^{\prime}\left(\left(y_{i}-\hat{\mu}_{1}[\tau]\right) / c s_{0}[\tau]\right)+\sum_{i=\tau+1}^{n} \psi^{\prime}\left(\left(y_{i}-\hat{\mu}_{2}[\tau]\right) / c s_{0}[\tau]\right)\right|}
$$

Differentiating $\psi$ in Equation (16) to obtain $\psi^{\prime}$, and that standard deviation of the error is acquired based on asymptotic variance for the M-estimators of location. [15] called the scale estimator in Equation (15) as A-estimator which seems to perform satisfactorily, and yet possesses good features among others robust scale estimators.

The bisquare function is adopted in Equation (15) that is defined as

$$
\psi(u)= \begin{cases}u\left(1-u^{2}\right)^{2}, & |u| \leq 1, \\ 0, & |u|>1 .\end{cases}
$$

In a sense, the function simply implies that measurements further away from the center will be gradually downweighted, and those fall beyond $c s_{0}[\tau]$ are completely eliminated. The tuning constant $c=9$ is chosen because of the reasonably well performed from extensive numerical simulations. By means of Equation (15), $\hat{\tau}$ is obtained by

$$
\hat{\tau}=\arg \min \{\log (\hat{\sigma}[\tau])\} .
$$

Having detected the shift, the null-model Equation (6) is tested against the alternative model Equation (7). The test statistic is given by

$$
R T=\sqrt{\frac{\hat{\tau}(n-\hat{\tau})}{n}}\left(\frac{\hat{\mu}_{2}-\hat{\mu}_{1}}{\hat{\sigma}[\hat{\tau}]}\right) .
$$

To decide whether or not to reject the null hypothesis $H$, it is crucial to determine the critical values of the suggested test statistics. From simulations, it was demonstrated that the distribution of $R T^{2} / n_{1}$ can be approximated by an F-distribution, with $n_{1}$ and $n_{2}$ degrees of freedom for a particular sample size $n$. Further details on the computation of the critical values as well as $n_{1}$ and $n_{2}$ can be found in [13].

Once the shift has been located, the observations will be divided into two groups $y_{1}, y_{2}, \ldots, y_{\tau}$ and $y_{\tau+1}, y_{\tau+2}, \ldots, y_{n}$. We then employ the similar framework to both groups in order to verify whether or not there are more shifts available. More succinctly, the framework is depicted as follows:

- Upon the detected shift, the observations are divided into two groups $y_{1}, y_{2}, \ldots, y_{\tau}$ and $y_{\tau+1}, y_{\tau+2}, \ldots, y_{n}$.

- The preceding framework is then employed to both groups, justifying if there are any additional shifts, or when the sample size is less than 4 or any specifically selected value.

In the case of multiple shifts, let $\hat{\mu}_{1}, \hat{\mu}_{2}, \ldots, \hat{\mu}_{k}$ be the computed means of the groups for located shifts $\hat{\tau}_{2}, \hat{\tau}_{3}, \ldots, \hat{\tau}_{k}$, the Equation (19) is then re-evaluated by 


$$
\hat{\sigma}=\frac{\left.n\left(c s_{0}\right) \mid \begin{array}{l}
\sum_{i=1}^{\hat{t}_{1}} \psi^{2}\left(\left(y_{i}-\hat{\mu}_{1}\right) / c s_{0}\right) \\
+\ldots+\sum_{i=\hat{\tau}_{k-1}+1}^{n} \psi^{2}\left(\left(y_{i}-\hat{\mu}_{k}\right) / c s_{0}\right)
\end{array}\right)^{1 / 2}}{\sqrt{n-k}\left|\begin{array}{l}
\sum_{i=1}^{\hat{t}_{1}} \psi^{\prime}\left(\left(y_{i}-\hat{\mu}_{1}\right) / c s_{0}\right) \\
+\ldots+\sum_{i=\hat{\tau}_{k-1}+1}^{n} \psi\left(\left(y_{i}-\hat{\mu}_{k}\right) / c s_{0}\right)
\end{array}\right|} .
$$

where $s_{0}=$ median $\left\{\left|y_{i}-m_{j}\right|\right\}$, for $j=1,2, \ldots, k$ with $m_{j}$ being the median of subgroup $j$. To take into account for the possibility of the loss of degrees of freedom in estimating the $\mu_{1}, \mu_{2}, \ldots, \mu_{k}, \sqrt{n}$ as an numerator of Equation (15) is substituted by $\sqrt{n^{2}(n-k)}$. Each group $(j=1,2, \ldots, k)$ lower control limit (LCL) and upper control limit (UCL) for the proposed chart are now evaluated via

$$
\begin{gathered}
\mathrm{LCL}_{j}=\hat{\mu}_{j}-h \sqrt{\frac{\hat{\tau}_{j+1}-\hat{\tau}_{j}-1}{\hat{\tau}_{j+1}-\hat{\tau}_{j}}} \hat{\sigma}, \\
\mathrm{UCL}_{j}=\hat{\mu}_{j}+h \sqrt{\frac{\hat{\tau}_{j+1}-\hat{\tau}_{j}-1}{\hat{\tau}_{j+1}-\hat{\tau}_{j}}} \hat{\sigma} .
\end{gathered}
$$

Put it simply, let $\hat{\tau}_{1}=0$ and $\hat{\tau}_{k+1}=n, \sqrt{\frac{\hat{\tau}_{j+1}-\hat{\tau}_{j}-1}{\hat{\tau}_{j+1}-\hat{\tau}_{j}}}$ reflects the connection between $y_{i}$ and the control limits. The conventional value of $h=3$ is utilized to determine the amount of evidence needed to deem a particular value as an outlying observation.

\section{The proposed control-charting procedure}

Section 3 illustrated [13] charting procedure. In accordance to Equation (13), $s_{0}[\tau]$ is one of the components in the $\psi$ function, where $s_{0}[\tau]$ is estimated by using median version given by Equation (14). A little weakness of using median absolute deviation (MAD) is that even though MAD has high breakdown-point (50\%), it has low efficiency (37\%) ([16]). Hence, instead of using MAD estimator for $s_{0}[\tau]$ described by Equation (14), we propose using the modified $s_{0}[\tau]$ estimator indicated in Equation (21), incorporating a more efficient M-scale estimator which possesses 50\% high breakdown bound as described in Equation (1). To be specific, the newly proposed $s_{0}[\tau]$ is defined as

$$
s_{0}[\tau]=\operatorname{median}\left\{m_{1}, m_{2}\right\},
$$

where $m_{1}=m_{1}[\tau]$ for $1 \leq i \leq \tau, m_{2}=m_{2}[\tau]$ for $\tau+1 \leq i \leq n$, and $m_{1}[\tau]$ and $m_{2}[\tau]$ are the M-scale estimates of $y_{1}, y_{2}, \ldots, y_{\tau}$ and $y_{\tau+1}, y_{\tau+2}, . ., y_{n}$, respectively. Strictly speaking, M- 
scale estimators now appear to dominate the field due to their generality, as well as possessing high breakdown-point and efficiency [8].

However, the distribution of the proposed test statistic $R T$ in Equation (18) does not seem easily tractable. Consequently, the critical values were obtained from the distribution of test statistic in Equation (18) by simulations. For illustrations, the data were generated from a standard normal distribution and this involved 100,000 random samples of sizes 10, 20, 30 and 50 covering significance level $\alpha$ values of $0.025,0.05$ and 0.10 . The test statistic in Equation (18) via various sample sizes was repeated for 100,000 times under null-model of Equation (6), with that the corresponding desired percentile values were determined from the generated empirical distribution. Table 1 presents the simulated cut-off values for test statistics in Equation (18) with sample sizes of 10, 20, 30 and 50. Details about the determination of cut-off values for the MAD framework can be found in [13]. On the basis of the cut-off values obtained, in the case where the position shift has been estimated and tested, the analogous control charting framework as indicated in Section 3 is implemented until the respective pairs of control limits for each interval are determined using Equation (20).

Table 1. Simulated critical values using M-scale estimator for test statistic, $R T$ for different significance levels $\alpha$.

\begin{tabular}{cccc}
\hline \hline \multirow{3}{*}{$n$} & \multicolumn{3}{c}{ M-scale Method } \\
\cline { 2 - 4 } & $\alpha=0.025$ & $\alpha=0.05$ & $\alpha=0.10$ \\
\hline 10 & 4.42 & 3.55 & 2.88 \\
\hline 20 & 3.58 & 3.13 & 2.64 \\
\hline 30 & 3.43 & 3.03 & 2.61 \\
\hline 50 & 3.33 & 3.02 & 2.60 \\
\hline \hline
\end{tabular}

\section{Illustrative example: application to wood moisture content data}

For verification, in this section we intend to apply the framework of the proposed approach by analyzing a real data set. The data was obtained from a furniture company in Malaysia in which the wood moisture content measures are the quality characteristic of interest. Here there were 37 subgroups under consideration, and the values of moisture content were measured at eight different parts for each of the furniture. This implies that the data set consist of 37 subgroups each of size 8 . To reflect the individual measurements, the associated chart was constructed by averaging the eight measurements for each of 37 subgroups.

To gain insight into the motivation for the approach in exploratory context, the conventional $X$-bar $(\bar{X})$ control chart is employed using the subgroups measurements for comparison purposes. The control limits are constructed through the within-group estimates using the average range and the chart is presented in Fig. 2. It can be seen that the control chart locates two signals that imply the evidence of between-groups variation which cannot be explained by the within-groups variation. For such behavior, the two signals would indicate that the wood moisture contents are not completely in its steady state in the sample. Perhaps it may offer some hints and yet not definite enough to distinguish the assignable cause. Moreover, the $\bar{X}$ chart locates some large outlying values but does not identify the existence of the possible shifts. A quite large standard deviation of the in-control process is estimated to be 1.429 . This could be accounted by the reason that there is an inclusion of extra variation due to the shifts. In addition, there appears to be that $\bar{X}$ chart is not as sturdy as the M-estimator being applied in the proposed method. Evidently, the chart is able to indicate the shifts, but it does not explicitly indicate about their numbers or the time points 
where they happen. Without incorporating the located shifts in the analysis, the chart seems to be less competent to detect the rest of the assignable causes.

For comparison purposes, Fig. 3 and Fig. 4 display the plots for the wood moisture content measurements by means of the procedure using the existing MAD and the proposed M-scale estimators. The upper and lower control limits are computed by adopting Equation (20). From the figures, both control charts are able to discover the existence of few assignable causes, one of which is the isolated disturbance located by sample number 34 , along with the two shifts in mean. After eliminating the outliers and rectifying the estimations caused by these outliers, the distribution is now can be explained satisfactorily by a normal distribution. In which case the standard deviation of M-scale method is slightly less than MAD method, which are respectively 0.6116 and 0.6286 . This may be due to that the effects of outlying data values had been eliminated.

Another key aspect in judging the performance of chart is how quickly it responds or reacts to the change in a process. In this instance, the control charts in Fig. 3 and Fig. 4 give the significant suggestions of their nature and the time points on which they have manifested. These plots reveal that the content averages are actually shifted between sample 5 and sample 6; sample 27 and sample 28. Apart from the indicated shifts, the content averages seem to be in statistical control, with no assignable causes appear to come from the remaining observations.

In the event that if the inquirer is not intended to know whether the additional variation is due to between-groups variation, but merely to check if the extra variation is distributed randomly, then the average moving range (AMR)-chart can be plotted. This is shown in Fig. 5 , with averages of wood moisture content served as individual observations. The chart of this kind is normally utilized in individuals control chart [17]. It is quite apparent that the chart fails to discover the shift in mean. Hence, as a whole, it appears to suggest that the procedure utilizing M-scale estimator has demonstrated the most convincing performance in terms its variation property for this data set.

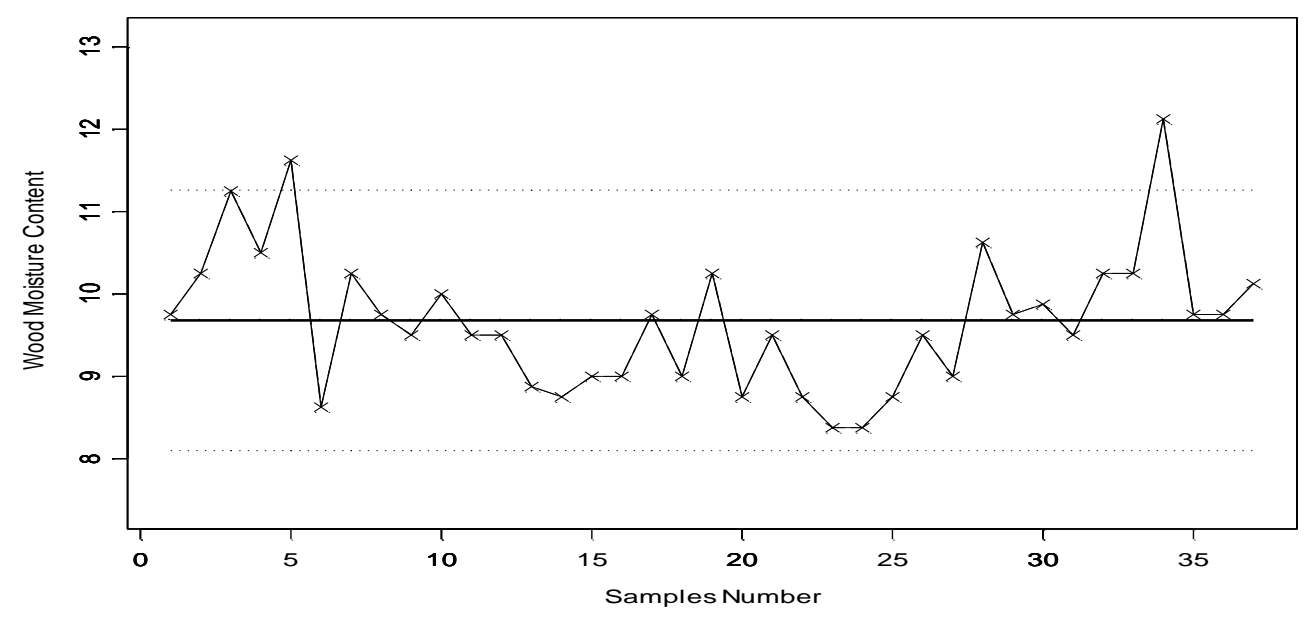

Fig. 2. $X$-bar control chart for wood moisture content. 


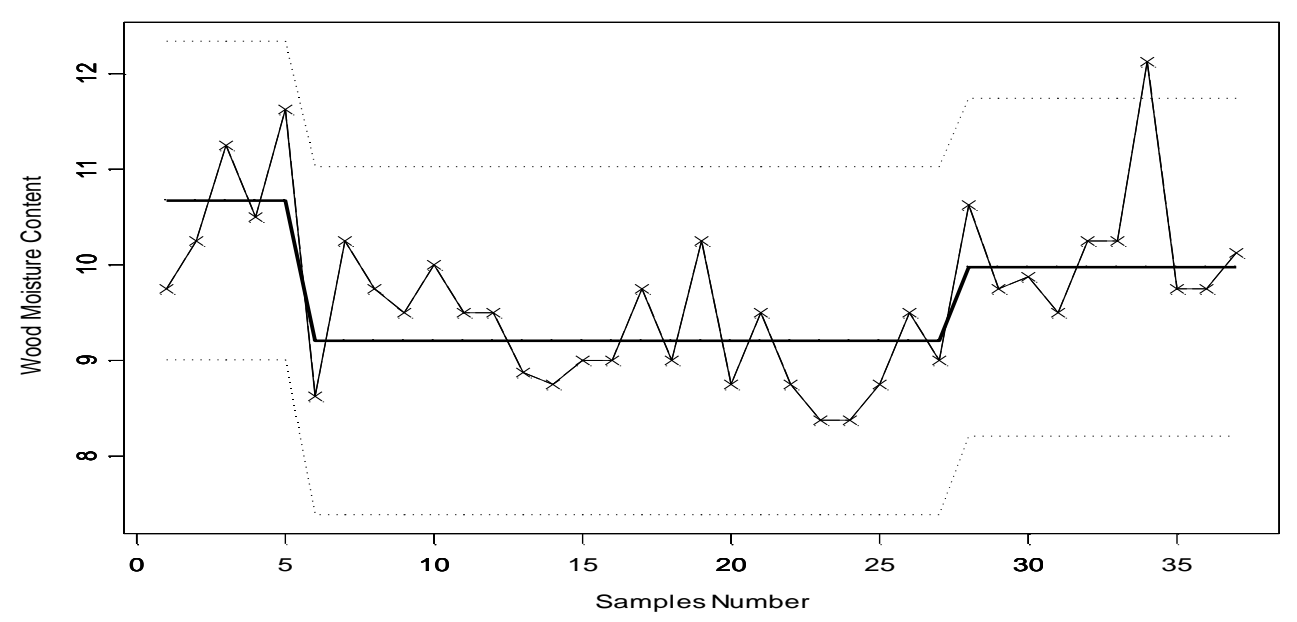

Fig. 3. Robust individuals control chart for wood moisture content using MAD estimator.

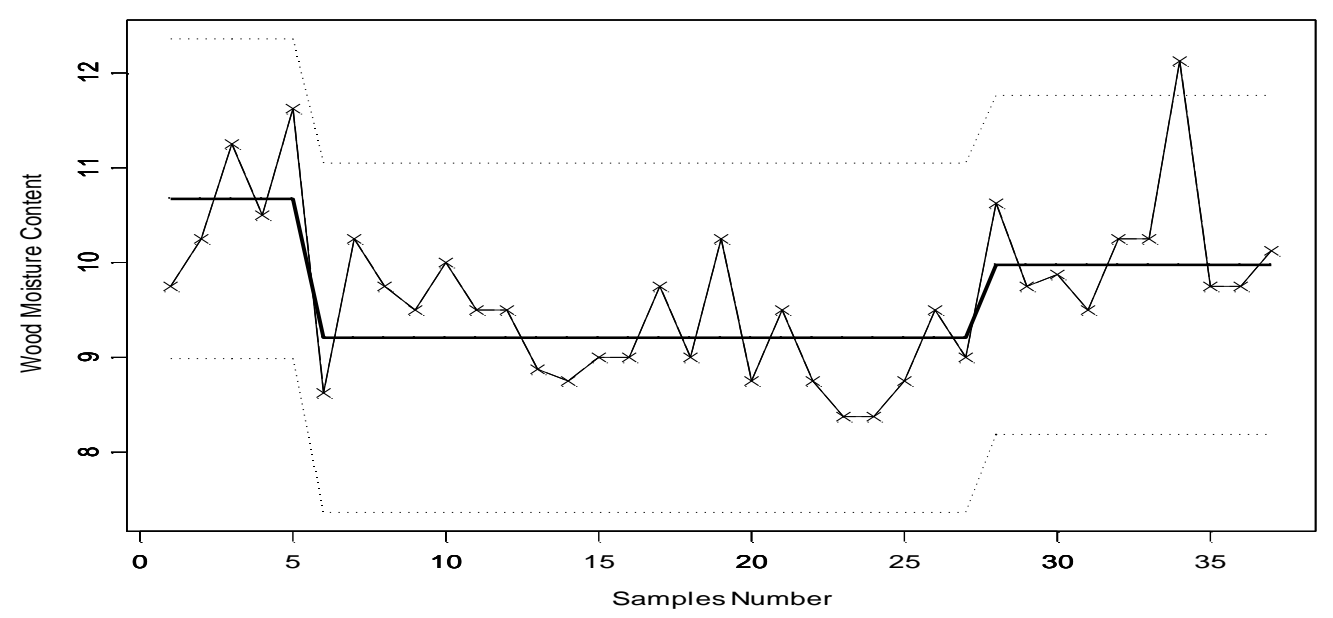

Fig. 4. The proposed robust individuals control chart for wood moisture content using M-scale estimator. 


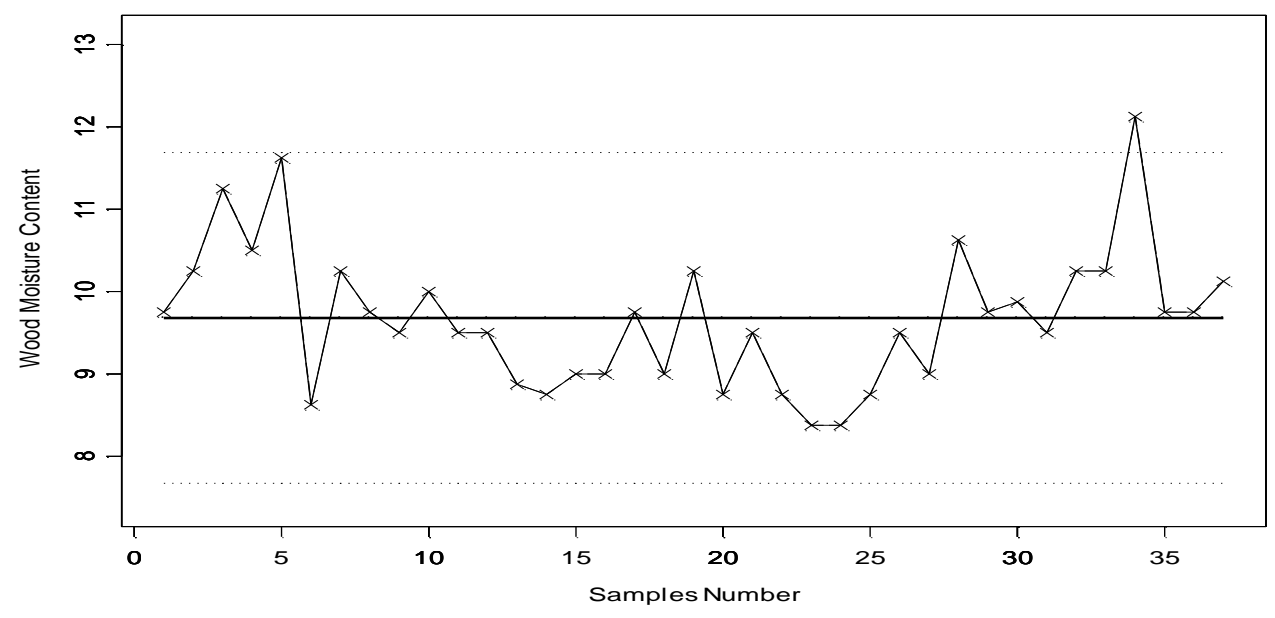

Fig. 5. AMR control chart for wood moisture content.

\section{Conclusion}

In this paper, we had demonstrated the use of so-called exploratory data analysis robust modified individuals control chart incorporating the $\mathrm{M}$-scale estimator and had made some comparisons to the existing charts. In which case, the proposed modified chart indeed offers relatively notable advantages over traditional control charts. It has been attempting to present an alternative robust control chart for detecting the process shift and variability for individual measurements in phase I, with the required table of critical values for testing the significance of the shift. The proposed modified robust individuals control chart which incorporates the M-scale estimator in order to compute the process standard deviation $\sigma$ offers substantial improvements over the existing MAD framework. With respect to the application in real data set, the proposed approach seems to perform marginally better than MAD method and outperforms other conventional charts in the presence of contamination. Thus, it is for these reasons that the proposed modified robust individuals control chart is preferred especially when there is a possible existence of outliers in data collection process.

The authors would like to thank Universiti Tunku Abdul Rahman, UTAR, for the financial assistance. The authors also would like express gratitude to Malaysia Ministry of Education. This research was supported by grant from the Malaysia Fundamental Research Grant Scheme (FRGS) (Grant No.: R/FRGS/A0700/01450A/001/2018/00560).

\section{References}

1. B.C. Khoo, Qual. Eng. 17, 243 - 257 (2005)

2. D.C. Hoaglin, F. Mosteller, J.W. Tukey, Understanding robust and exploratory data analysis (Wiley: New York, 1983)

3. P. Fearnhead, G. Rigaill, J. Am. Stat. Assoc. 114, 169 - 183 (2019)

4. C. Zhang, N. Chen, C.L. Zou, J. Qual. Technol. 48, 139 - 161 (2016)

5. W.J. Liang, D.D. Xiang, X.L. Pu, J. Qual. Technol. 48, 265 - 283 (2016)

6. H.Z. Nazir, M. Schoonhoven, M. Riaz, R.J.M.M. Does, Qual. Eng. 26, 246 - 252 (2014) 
7. M. Atalay, M.C. Testik, S. Duran, C.H. Weiß, Qual. Eng. 32, 223 - 243 (2020)

8. P.J. Huber, Robust statistics (Wiley, New York, 1981)

9. F.R. Hampel, E.M. Ronchetti, P.J. Rousseew, W.A. Stahel, Robust statistics: the approach based on influence functions (Wiley, New York,1986)

10. V.J. Yohai, Ann. Stat. 15, 642 - 656 (1987)

11. F.R. Hampel, J. Am. Stat. Assoc. 69, 383 - 93 (1974)

12. P.J. Rousseeuw, A.M. Leroy, Robust regression and outlier detection (1987)

13. J. De Mast, K.C.B. Roes, Qual. Eng. 16, 407 - 421 (2004)

14. J.H. Sullivan, W.H. Woodall, J. Qual. Technol. 28, 265 - 278 (1996)

15. D.A. Lax, J. Am. Stat. Assoc. 80, 736 - 741 (1985)

16. M.O.A. Abu-Shawiesh, Int. J. Qual. Reliab. Manag. 26, 480 - 496 (2008)

17. K.C.B. Roes, R.J.M.M. Does, Y. Schurink, J. Qual. Technol. 25, 188 - 198 (1993) 